\title{
Magnetic Study of the Fe Coated by Au Nanoparticles
}

\author{
A. ZELEŇÁKOVÁ
}

Dept. of Solid State Physics, UPJŠ, Park Angelinum 9, 04154 Košice, Slovakia

$$
\text { J. KováČ, V. KaveČAnskÝ }
$$

Institute of Exp. Physics, SAS, Park Angelinum 9, 04001 Košice, Slovakia

$$
\text { AND V. ZELEŇÁK }
$$

Dept. of Inorganic Chemistry, UPJŠ, Moyzesova 51, 04001 Košice, Slovakia

Nanosized iron-gold magnetic nanoparticles with an average particle size $10 \mathrm{~nm}$ were prepared by a reverse micelle method. The magnetic properties measurements of DC and AC magnetization confirm behaviour typical of a superparamagnetic system, such as the irreversibility of the zero-field-cooled and field-cooled curves, the frequency dependence of a blocking temperature $T_{\mathrm{B}}$, and revealing of coercivity $H_{\mathrm{C}}$ below blocking temperature. The quantitative analysis of $\mathrm{AC}$ susceptibility due to value of parameter $C_{1}=\Delta T_{\mathrm{B}} /\left(T_{\mathrm{B}} \Delta \log f\right)=0.0242$ confirming the existence of inter-particle interaction in our system.

PACS numbers: 61.46.Df, 68.37.Lp, 75.20.--g, 75.30.Cr

\section{Introduction}

Magnetic nanoparticles have attracted considerable interest due to their significance in technological applications as well as for the fundamental physics $[1,2]$. Below a critical size of particles, the magnetic moment in single domain fluctuates in direction due to thermal agitation, leading to superparamagnetic behaviour above the blocking temperature $T_{\mathrm{B}}$, and to spatial freezing of these moments below $T_{\mathrm{B}}[3]$.

In the present work, the nanoparticles of iron coated by gold $(\mathrm{Fe} / \mathrm{Au})$ were prepared by chemical method and their magnetic characteristics were analysed in the content of theoretical laws (Langevin function, Arrhenius law and VogelFulcher law) [3-5]. 


\section{Experimental}

Nanoparticles of $\mathrm{Fe} / \mathrm{Au}$ were prepared using reverse micelle method with cetyltrimethylammonium bromide $(\mathrm{CTAB})$ as the surfactant and with octane as the oil phase [5]. Aqueous solutions of $\mathrm{FeSO}_{4}, \mathrm{NaBH}_{4}$, and $\mathrm{HAuCl}_{4}$ were used during synthesis. The ferromagnetic iron formed a core of the nanoparticles while the diamagnetic gold a shell of the core. The structure and the microstructure of the nanoparticles was studied by X-ray diffraction (XRD) using a powder diffractometer and with transmission electron microscope (TEM). Magnetic measurements were performed on a commercial SQUID-based magnetometer (Quantum Design) over a wide range of temperatures $(2-300 \mathrm{~K})$ and applied DC fields (up to $5 \mathrm{kOe}$ ). The same instrument was used for AC susceptibility measurements carried out at frequencies $1-500 \mathrm{~Hz}$ in a temperature region encompassing the blocking temperatures.

\section{Results and discussion}

TEM observation showed that the Fe/Au nanoparticles are isolated without aggregation and of clearly spherical shape with average size $10 \mathrm{~nm}$ [5]. The X-ray diffraction pattern of $\mathrm{Fe} / \mathrm{Au}$ nanoparticles is presented in Fig. 1.

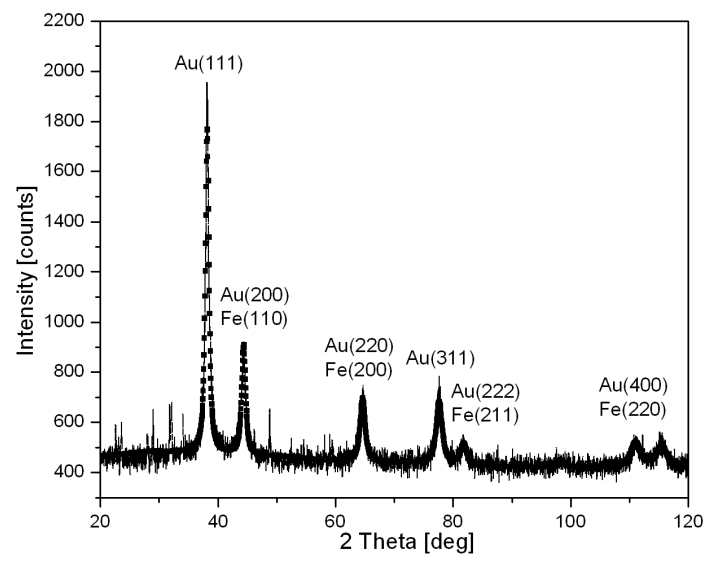

Fig. 1. X-ray diffraction pattern of the Fe/Au nanoparticles.

The major peaks are corresponding to the fcc metallic gold diffraction. The XRD pattern of bcc $(\alpha)$ iron is hidden under the pattern of fcc gold due to the overlapping of their diffraction peaks. Although diffraction studies cannot differentiate between iron and gold in the system, they do demonstrate and confirm a high degree of crystallinity and uniformity in the particles.

The zero-field-cooled (ZFC) and field-cooled (FC) magnetisation curves measured in the DC field of 5000 Oe, Fig. 2a, exhibit the irreversibility and narrow maximum of $\mathrm{ZFC}$ at the peak value of $T_{\mathrm{B}} \approx 20 \mathrm{~K}$. This indicates that below 
blocking temperature $T_{\mathrm{B}}$, the magnetic moment of each particle is blocked along its easy magnetisation axis, whereas above $T_{\mathrm{B}}$ the particles are characterised by superparamagnetic behaviour. The shape of the ZFC curve also suggests a relatively narrow size distribution of the $\mathrm{Fe} / \mathrm{Au}$ nanoparticles. The field dependence of magnetisation, Fig. 2b, measured at the temperatures $2 \mathrm{~K}$ and $300 \mathrm{~K}$ confirms the paramagnetic behaviour of particles above $T_{\mathrm{B}}$ and appearing of coercivity below $T_{\mathrm{B}}$. Unusual high value of coercivity $H_{\mathrm{C}} \approx 2850$ Oe measured at $2 \mathrm{~K}$, and the shape of hysteresis loop can be caused by additional antiferromagnetic interaction probably due to a dipolar interaction among particles or due to interaction of particles in core/shell structure [5]. DC magnetic data were fitted by both Langevin function for non-interacting particles and modified Langevin function for interacting nanoparticles [2-6]. The analyses of fitted procedure confirm the superparamagnetic behaviour of interacting particles in our system. The value of magnetic moment of particle $\mathrm{Fe} / \mathrm{Au}$ estimated by fitting of $M(H)$ data was $m_{\mathrm{p}} \approx 49 \mu_{\mathrm{B}}$

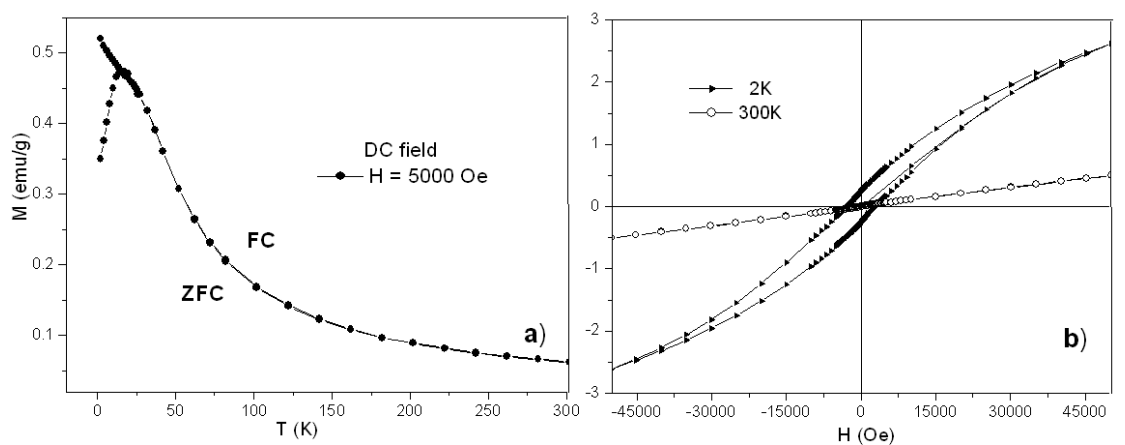

Fig. 2. Magnetic properties of Fe/Au measured in DC magnetic field: (a) temperature dependence of magnetisation, (b) field dependence of magnetisation.

To investigate the presence of inter-particle interaction, we performed $\mathrm{AC}$ susceptibility measurements at different frequencies $(1-500 \mathrm{~Hz})$ and DC applied fields (0-1000 Oe). It is evident from Fig. 3a that the real part of the AC susceptibility $\chi^{\prime}$ is frequency dependent and the peak position increases with increase in frequency. Also, the dependence in Fig. 3b shows that the peak of of AC susceptibility is affected by application of low DC fields: it broadens and shifts downwards. This is qualitative confirmation of blocking/freezing process in $\mathrm{Fe} / \mathrm{Au}$ nanoparticles system. The AC susceptibility data were fitted by both Arrhenius law valid for a system of non-interacting particles and by Vogel-Fulcher law describing system of interacting particles $[2-4,6]$. The quantitative analysis of $\chi^{\prime}$ due to the value of parameter $C_{1}=\Delta T_{\mathrm{B}} /\left(T_{\mathrm{B}} \Delta \log f\right)=0.00242$ confirms the existence of inter-particle interaction in our system. 


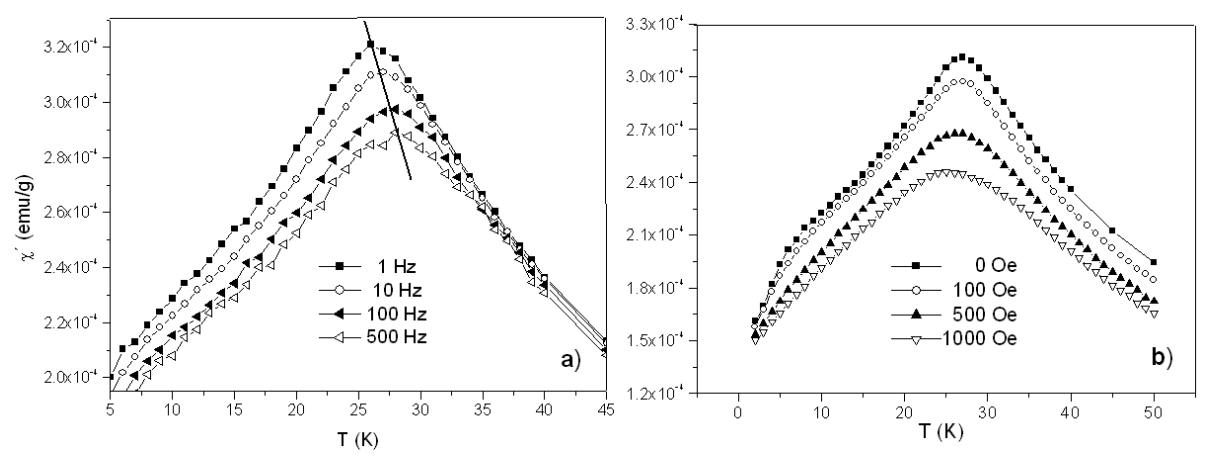

Fig. 3. Temperature dependence of real component of the AC susceptibility measured at different (a) frequencies (1-500 Hz), (b) DC applied fields (0-1000 Oe), measured at frequency of $10 \mathrm{~Hz}$.

\section{Conclusion}

The superparamagnetic Fe/Au spherical nanoparticles with $10 \mathrm{~nm}$ diameter were prepared. The blocking/freezing process was demonstrated by the DC and AC magnetization measurements. The qualitative analysis of measured data confirms the existence of inter-particle interaction in the system.

\section{Acknowledgments}

This work was supported by Science and Technology Assistance Agency under the contract No. APVV-20-008404, by the Scientific Grant Agency (VEGA 1/4020/07) and by VVGS UPJŠ 57/5/3/06.

\section{References}

[1] Y. Sun, M.B. Salamon, K. Garnier, R. S. Averback, Phys. Rev. Lett. 91, 167206 (2003).

[2] J.A. De Torro, M.A. de la Torre, J.M. Riveiro, R. Sáez Puche, A. Gómez-Herrero, L.C. Otero-Díaz, Phys. Rev. B 60, 12918 (1999).

[3] M. Tadic, D. Markovic, V. Spasojevic, V. Kusigerski, M. Remškar, J. Pirnat, Z. Jagličićt, J. Alloys Comp., in press.

[4] S.K. Sharma, R. Kumar, S. Kumar, V.V.S. Kumar, M. Knobel, V.R. Reddy, A. Banerjee, M. Singh, Solid State Commun. 141, 203 (2007).

[5] A. Kakay, W.M. Gutowski, L. Takacs, L.K. Varga, J. Phys. A 37, 6027 (2004).

[6] A. Zeleňáková, V. Zeleňák, J. Degmová, J. Kováč, K. Sedláčková, M. Kusý, J. Sitek, J. Alloys Comp., accepted for publication. 